\title{
A Comprehensive Study of Progressive Cytogenetic Alterations in Clear Cell Renal Cell Carcinoma and a New Model for ccRCC Tumorigenesis and Progression
}

\author{
Zhongfa Zhang, ${ }^{1,2}$ Bill Wondergem, ${ }^{1}$ and Karl Dykema ${ }^{3}$ \\ ${ }^{1}$ Laboratory of Cancer Genetics, Van Andel Research Institute, Grand Rapids, MI 49503, USA \\ ${ }^{2}$ Center for Systems and Computational Biology, The Wistar Institute, Philadelphia, PA 19104, USA \\ ${ }^{3}$ Computational Biology, Van Andel Research Institute, Grand Rapids, MI 49503, USA
}

Correspondence should be addressed to Zhongfa Zhang, zfzhang2@hotmail.com

Received 8 October 2009; Accepted 20 May 2010

Academic Editor: Muneesh Tewari

Copyright ( $) 2010$ Zhongfa Zhang et al. This is an open access article distributed under the Creative Commons Attribution License, which permits unrestricted use, distribution, and reproduction in any medium, provided the original work is properly cited.

\begin{abstract}
We present a comprehensive study of cytogenetic alterations that occur during the progression of clear cell renal cell carcinoma (ccRCC). We used high-density high-throughput Affymetrix $100 \mathrm{~K} \mathrm{SNP}$ arrays to obtain the whole genome SNP copy number information from 71 pretreatment tissue samples with RCC tumors; of those, 42 samples were of human ccRCC subtype. We analyzed patterns of cytogenetic loss and gain from different RCC subtypes and in particular, different stages and grades of ccRCC tumors, using a novel algorithm that we have designed. Based on patterns of cytogenetic alterations in chromosomal regions with frequent losses and gains, we inferred the involvement of candidate genes from these regions in ccRCC tumorigenesis and development. We then proposed a new model of ccRCC tumorigenesis and progression. Our study serves as a comprehensive overview of cytogenetic alterations in a collection of 572 ccRCC tumors from diversified studies and should facilitate the search for specific genes associated with the disease.
\end{abstract}

\section{Introduction}

Cytogenetic changes underlie most genetic diseases, including cancer. Not only are different patterns of cytogenetic alterations associated with different types of tumors, tumors of the same type at different stages of development also exhibit different cytogenetic patterns. It is commonly accepted that tumors at early stages of development have fewer cytogenetic alterations than tumors at more advanced stages. Early mutations in a few key genes are believed to drive the initial steps of tumorigenesis. Key mutational events are loss-of-function mutations in tumor suppressor genes (TSGs) and/or gain-of-function mutations in oncogenes. The resulting changes in gene function are thought to trigger the process of tumorigenesis and set the stage for the accumulation of more genetic abnormalities as the tumor progresses. Here we have treated gene methylation as a special form of mutation, for ease of description.
Mutations are rare. In the two-hit theory of cancer formation [1], loss of TSG function occurs in two stages: mutation of one of the two parental gene copies occurs as the first "hit", followed by a second hit when a large-orsmall-scale chromosomal deletion or a structural alteration inactivates the remaining TSG allele. Thus, TSGs are often contained in regions having copy number loss. On the other hand, an oncogene often gains its functionality via an increase in gene copy number or, in some cases, activating mutations of the gene. Therefore, an oncogene is more likely to be contained in regions where copy numbers of DNA sequences are amplified. Analysis of cytogenetic losses and gains in tumor cells can thus identify potential tumor suppressors and oncogenes.

Advances in technology now allow for cytogenetic analysis in unprecedented detail. Detailed cytogenetic analysis of a given tumor type at different stages of progression will be necessary for a full understanding of tumor development. 
Cytogenetic profiling of different tumor subtypes can also shed light on the understanding and diagnosis of cancer by revealing genetic alterations specific for each subtype. In this study, we undertook a comprehensive survey of cytogenetic losses and gains occurring in renal cell carcinoma (RCC) by using the high throughput high density Affymetrix $100 \mathrm{~K}$ SNP chips.

RCC is a heterogeneous disease consisting of multiple subtypes. The most common RCC subtype is clear cell (ccRCC), accounting for about $70 \%$ of all RCC tumors. Other subtypes include papillary (PA, 10\%), chromophobe $(\mathrm{CH}, \sim 5 \%)$, oncocytoma (ON, $<1 \%)$, and collecting duct carcinomas (very rare) $[2,3]$. Despite large-scale genomic screening, the only gene that is commonly mutated is the von Hippel-Lindau (VHL) gene [4]. Mutations, methylation, and/or LOH of the VHL gene are frequently observed in ccRCC tumors ( $90 \%)$. Cytogenetic profiling of various RCC subtypes has been studied indirectly through genomewide gene expression profiling $[2,5,6]$. Traditional methods such as comparative genomic hybridization (CGH) [7-12], quantitative PCR [13], fluorescent in situ hybridization (FISH), and cytogenetic banding [14, 15] have also been applied. Only recently has the use of high-throughput high density DNA microarray chips become available for detailed genome-wide cytogenetic alteration study. These microarrays offer higher resolution than traditional methods. For example, we recently used such high density microarrays to profile genome-wide cytogenetic alterations in ccRCC and papillary RCC [16] and Beroukhim et al. combined both $500 \mathrm{~K}$ SNP data and gene expression data to identify the cytogenetic alterations in VHL and sporadic tumors [17]. However, little is known about the specific sequence by which genetic alterations take place during ccRCC progression. The most relevant study to our current one is from Jiang et al., who relied on comparative genomic hybridization data to construct an evolutionary tree model for RCC [18]. To our knowledge, there have been no systematic studies characterizing progressive cytogenetic alterations in ccRCC development using high-throughput high-density 100K SNP microarray data.

Here we report a systematic study of cytogenetic alteration profiles in ccRCC tumors at different stages of development and in association with tumor grades. Our study provides a detailed map of the sequence of cytogenetic alterations that occur during the progression of ccRCC. Based on these results, we propose a new model of ccRCC tumor genesis and progression. Our results suggest the involvement of cytogenetic alterations seen in ccRCC, and will aid in the identification of genes relevant to ccRCC.

\section{Material and Methods}

2.1. Tissue Collection. 71 pretreatment tissue samples were collected from multiple cancer centers, including Spectrum Health Hospital in Grand Rapids, MI (31 cases), and the Cooperative Human Tissue Network (CHTN, 38 cases) over the past few years. There were 42 cases of ccRCC, 6 cases of chromophobe, 8 cases of oncocytoma, and 15 cases of papillary RCC. Clinicopathological information for the 42 ccRCC patients was summarized in Table 1 . All tumors were primary and sporadic in that the patients did not have a known familial history of their tumor types. The tissues were pulverized under liquid nitrogen and genomic DNA was prepared by a standard protocol using proteinase K/SDS lysis followed by phenol/chloroform extraction and ethanol precipitation. The clinicopathological diagnosis of tumors followed the standard TNM diagnosis of RCC. Tumor staging and grading as well as other pathological factors (e.g., vein or vascular invasions, necrosis or sarcomatoid factors, etc.) were obtained from review of the pathology staining reports and evaluation of the case notes by individual clinicians. In particular, the difference between $\mathrm{T} 1 \mathrm{a}$ and $\mathrm{T} 1 \mathrm{~b}$ tumors is largely in the tumor size. Readers can refer to the American Cancer Society for more information on tumor staging criteria (http://www.cancer.org/). VHL mutations were obtained through sequencing of all three exons of the gene.

In addition to our current study, we also selected 5 largescale cytogenetic studies in the literature for a combined study. Out of the 5 studies, 3 had tumor stage information, 2 did not. There were 572 ccRCC samples in total with different techniques used to detect cytogenetic alterations. The clinicopathological information was summarized in Table 1. The copy number analysis based on tumor stages was summarized in Table 2.

\subsection{DNA Preparation. We used Affymetrix $100 \mathrm{~K}$ SNP chips to obtain genomic information. Each $100 \mathrm{~K}$ SNP chip contains two subchips using either XbaI or HindIII to digest the genome materials. Digestion of the DNA, PCR, and scanning followed the instructions from Affymetrix (http://www.affymetrix.com/). All data samples passed the quality controls both before application to the chip and after scanning the hybridized chip.}

2.3. SNP Data Analysis. Raw SNP copy numbers were calculated based on CNAG software version 2 [21]. We used a total of 56 normal samples to obtain the normal copy number 2 signal. Among them, 48 were downloaded from Affymetrix and 8 were obtained from our own scans on normal kidney tissues that we have collected. The raw copy numbers were replaced by running $t$-test statistics (with a window size of 31 SNPs for the moving windows). The $t$-test makes use of the copy number's local constant property, which assumes that the losses or gains of SNP copy numbers tend to happen in segments. Neighboring SNPs tend to have the same copy numbers. Thus, by borrowing the information from its neighbors, this copy number calculation will increase its power of uncovering the underlying DNA alterations and decrease noise. This operation is equivalent to smoothing the calculated raw copy numbers. The correction is necessary because DNA samples of tumor tissues prepared for SNP runs were generally not a homogenous collection of tumor cells, but rather a mixed collection of tumor and other cells (e.g., stroma). Even the tumor cells may have different cytogenetic patterns with 
TABLE 1: Summary of clinicopathological characteristics of ccRCC samples.

\begin{tabular}{|c|c|c|c|c|c|c|c|c|}
\hline \multirow[b]{2}{*}{ Studies } & \multirow{2}{*}{\multicolumn{2}{|c|}{ Current }} & \multicolumn{5}{|c|}{ \#Samples } & \multirow[b]{2}{*}{ Total } \\
\hline & & & $\begin{array}{l}\text { Katte et al. } 09 \\
{[14]}\end{array}$ & $\begin{array}{l}\text { Beroud et al. } 96 \\
\text { [13] }\end{array}$ & $\begin{array}{l}\text { Gunawan et al. } \\
01[15]\end{array}$ & $\begin{array}{l}\text { Toma et al. } 08 \\
\text { [19] }\end{array}$ & $\begin{array}{l}\text { Yoshimoto et al. } \\
07[20]\end{array}$ & \\
\hline Study Size & 42 & & 246 & 118 & 118 & 22 & 26 & 572 \\
\hline \multicolumn{9}{|l|}{ Gender } \\
\hline $\mathrm{M}$ & 14 & & 170 & 81 & 60 & 13 & 21 & 359 \\
\hline $\mathrm{F}$ & 22 & & 76 & 37 & 58 & 9 & 5 & 207 \\
\hline Total & 36 & & 246 & 118 & 118 & 22 & 26 & 566 \\
\hline \multicolumn{9}{|l|}{ Age (years) } \\
\hline median & 64.5 & & 60.4 & 62 & 64 & 59 & 25 & 62 \\
\hline range & $39-85$ & & $24-86$ & $26-82$ & $32-81$ & $35-80$ & $46-85$ & $24-86$ \\
\hline \multicolumn{9}{|l|}{ Stage } \\
\hline pT1a & 10 & pT1 & 121 & 77 & 49 & 9 & 19 & \\
\hline $\mathrm{pT} 1 \mathrm{~b}+\mathrm{T} 2$ & 12 & pT2 & 25 & 14 & 12 & 7 & 2 & \\
\hline pT3a & 9 & pT3 & 97 & 22 & 34 & 5 & 2 & \\
\hline $\mathrm{pT} 3 \mathrm{~b}+\mathrm{T} 4$ & 7 & pT4 & 3 & 5 & 23 & 1 & 2 & \\
\hline Total & 38 & & 246 & 118 & 118 & 22 & 25 & 567 \\
\hline \multicolumn{9}{|l|}{ Fuhrman Grade } \\
\hline 1 & 3 & & & & & & & \\
\hline 2 & 12 & I & 29 & 13 & 42 & 2 & 2 & \\
\hline $2-3$ & 5 & II & 106 & 61 & 63 & 15 & 9 & \\
\hline 3 & 10 & III & 84 & 33 & 12 & 5 & 10 & \\
\hline $3-4$ & 8 & IV & 27 & 11 & 0 & 0 & 5 & \\
\hline 4 & 2 & & & & & & & \\
\hline Total & 40 & & 246 & 118 & 117 & 22 & 26 & 569 \\
\hline \multicolumn{9}{|l|}{ Size $(\mathrm{cm})$} \\
\hline median & 5 & & 6.6 & 6.6 & 6.67 & NA & NA & 6.6 \\
\hline range & $1.1-12.5$ & & $1-19$ & $1-16$ & $1.5-25$ & NA & NA & $1-25$ \\
\hline
\end{tabular}

NA: data not available.

differed combinations of numbers of signal for the target over signals for the control (such as $3: 2,2: 3$, etc.). For example, the low-grade and early-stage tumors tend to have a lower proportion of tumor cells than the high-grade and later-stage tumors. We call this phenomenon partial gain or partial loss, as opposed to complete gain or loss in that all cells used for microarray scan are tumor cells. As a result, without any corrections, the final raw copy numbers of the tumor cells would be a weighted mixture distribution of all the components of cells prepared for the scan, usually pulled toward the normal copy number of 2 . A solid cutoff to claim a gain or loss will be improper if one takes this into consideration.

2.4. Statistical Methods. The smoothed copy numbers were then summarized based on cytoband using the regional expression bias ( $\mathrm{reb}$ ) package in Bioconductor [22], adapted to the SNP data. Briefly, the algorithm grouped the probes by the associated SNP locations; for each region, a general test (such as binomial or $t$-test) was applied to determine if the raw copy numbers in the region were collectively higher or lower than that of normal. The test statistics were then output for each tumor sample and for each cytogenetic region. Disease-specific survival was used and was defined to be the time from first operation date to either death or last known follow-up date. For each cytogenetic region with cross sample interquartile range of the summarized cytogenetic alteration scores (the scores were the output from algorithm reb for each tumor and for each cytogenetic region) greater than 2.5, a number of survival models were built to associate the patients' survival with the summarized cytogenetic alteration scores. We set the number of models to be 100 for each cytoband. Each model was built on a randomly selected but fixed number of subset of ccRCC patients. The score test was used to calculate the prognostic significance of $P$-values for the association. The transformed $P$-values $(-\log 10(p))$ were averaged over that from the 100 models as the final significance of association for the cytoband, as well as the regression coefficients. A studentized test was used to test the difference of summarized cytogenetic alterations between two groups of samples.

2.5. Display of Copy Number Gains or Losses for a Group of Samples through Boxplot. To display the collective copy 
TABLE 2: Selected large-scale cytogenetic studies of ccRCC tumors.

\begin{tabular}{|c|c|c|c|c|c|c|c|c|c|}
\hline \multirow{3}{*}{\multicolumn{2}{|c|}{$\begin{array}{c}\text { Studies } \\
\text { Methods } \\
\text { \# samples }\end{array}$}} & & $\begin{array}{l}\text { Klatte et al. } \\
09\end{array}$ & $\begin{array}{l}\text { Beroud et al. } \\
96\end{array}$ & $\begin{array}{l}\text { Gunawan et al. } \\
01\end{array}$ & $\begin{array}{l}\text { Toma et al. } \\
08\end{array}$ & $\begin{array}{l}\text { Yoshimoto et al. } \\
07\end{array}$ & Current & Total \\
\hline & & & GPG & qPCR & G-Banding & SNP10K & BACPAC & SNP100K & \\
\hline & & & 246 & 118 (cc only) & 118 & 22 & 26 & 42 & 572 \\
\hline Event & & Stage & \multicolumn{7}{|c|}{ Number of events/Total Number of samples = percent } \\
\hline \multirow{5}{*}{$-3 p$} & & S1 & $77 / 121=64 \%$ & $52 / 77=68 \%$ & $48 / 49=98 \%$ & & & $19 / 22=86 \%$ & $\begin{array}{l}196 / 269= \\
73 \%\end{array}$ \\
\hline & & S2 & $17 / 25=68 \%$ & $10 / 14=71 \%$ & $12 / 12=100 \%$ & & & $2 / 2=100 \%$ & $41 / 53=77 \%$ \\
\hline & & S3 & $53 / 97=55 \%$ & $15 / 22=68 \%$ & $33 / 34=97 \%$ & & & $14 / 15=93 \%$ & $\begin{array}{l}115 / 168= \\
68 \%\end{array}$ \\
\hline & & S4 & $0 / 3=0 \%$ & $2 / 5=40 \%$ & $23 / 23=100 \%$ & & & $1 / 1=100 \%$ & $26 / 32=81 \%$ \\
\hline & & Sum & $\begin{array}{l}147 / 246= \\
60 \%\end{array}$ & $79 / 118=67 \%$ & $116 / 118=98 \%$ & $\begin{array}{l}20 / 22= \\
91 \%\end{array}$ & $21 / 26=81 \%$ & $36 / 40=90 \%$ & $\begin{array}{l}419 / 570= \\
74 \%\end{array}$ \\
\hline \multirow{5}{*}{$+5 q$} & & S1 & & & $28 / 49=57 \%$ & & & $10 / 22=45 \%$ & $38 / 71=54 \%$ \\
\hline & & S2 & & & $6 / 12=50 \%$ & & & $2 / 2=100 \%$ & $8 / 14=57 \%$ \\
\hline & & S3 & & & $23 / 34=67 \%$ & & & $7 / 15=47 \%$ & $30 / 49=61 \%$ \\
\hline & & S4 & & & $10 / 23=43 \%$ & & & $0 / 1=0 \%$ & $10 / 24=42 \%$ \\
\hline & & Sum & $82 / 246=33 \%$ & & $67 / 118=57 \%$ & $\begin{array}{l}10 / 22= \\
45 \%\end{array}$ & $15 / 26=58 \%$ & $19 / 40=47 \%$ & $\begin{array}{l}193 / 452= \\
43 \%\end{array}$ \\
\hline \multirow{5}{*}{$-14 q$} & & S1 & $24 / 121=20 \%$ & $19 / 77=25 \%$ & $29 / 49=59 \%$ & & & $5 / 22=23 \%$ & $77 / 269=29 \%$ \\
\hline & & S2 & $8 / 25=32 \%$ & $4 / 14=29 \%$ & $9 / 12=75 \%$ & & & $0 / 2=0 \%$ & $21 / 53=40 \%$ \\
\hline & & S3 & $36 / 97=37 \%$ & $8 / 22=37 \%$ & $22 / 34=65 \%$ & & & $7 / 15=47 \%$ & $73 / 168=43 \%$ \\
\hline & & S4 & $0 / 3=0 \%$ & $3 / 5=60 \%$ & $14 / 23=61 \%$ & & & $0 / 1=0 \%$ & $17 / 32=53 \%$ \\
\hline & & Sum & $68 / 246=28 \%$ & $34 / 118=29 \%$ & $74 / 118=63 \%$ & $\begin{array}{l}8 / 22= \\
36 \%\end{array}$ & $9 / 26=35 \%$ & $12 / 40=30 \%$ & $\begin{array}{l}205 / 570= \\
36 \%\end{array}$ \\
\hline+7 & & & $64 / 246=26 \%$ & & $22 / 118=19 \%$ & $\begin{array}{l}7 / 22= \\
32 \%\end{array}$ & $9 / 26=35 \%$ & $17 / 40=42 \%$ & $\begin{array}{l}119 / 452= \\
26 \%\end{array}$ \\
\hline$-8 \mathrm{p}$ & & & $49 / 246=20 \%$ & & $39 / 118=34 \%$ & & & $10 / 40=25 \%$ & $98 / 404=24 \%$ \\
\hline$-6 \mathrm{q}$ & & & $42 / 246=17 \%$ & & $28 / 118=24 \%$ & $\begin{array}{l}6 / 22= \\
27 \%\end{array}$ & $8 / 26=31 \%$ & $7 / 40=17 \%$ & $91 / 452=20 \%$ \\
\hline$-9 p$ & & & $40 / 246=19 \%$ & & $28 / 118=24 \%$ & $\begin{array}{l}7 / 22= \\
32 \%\end{array}$ & $5 / 26=19 \%$ & $7 / 40=18 \%$ & $87 / 452=19 \%$ \\
\hline$-4 p$ & & & $32 / 246=15 \%$ & & $17 / 118=14 \%$ & & & $2 / 40=5 \%$ & $51 / 404=13 \%$ \\
\hline
\end{tabular}

number gains or losses for a group of samples, we first calculated for each sample and for each cytoband, the summarization scores of copy numbers of SNPs within the cytoband through reb algorithm, which was described previously on the smoothed copy numbers. A positive value represents a gain, while a negative value represents a loss for the cytoband. The absolute values represent the degrees of losses or gains. The data from each cytoband over all samples in the group were then summarized and a box was produced to represent the summarization. The boxes were placed side by side ordered by their physical positions on the chromosome, from $\mathrm{p}$-arm to $\mathrm{q}$-arm, from chromosome 1 to 22. We omitted analysis for chromosome X. The upper and the lower bounds of the boxes are the third and first quartiles of the data sequences. If the box is well below zero, it indicates that the majority of the samples in the group have losses in their copy numbers in the cytoband (at least 75\%). If the box is well above zero, it is interpreted as majority of the samples in the group have gains in their copy numbers (at least 75\% again). Thus the boxes contain both the frequency and the intensity of the gain or loss events in the group. It is a natural combination of information for both. The vertical length of the box reflects the range of sample CNA (cytogenetic alteration numbers) values: the longer the box, the more variable the range of sample values. Thus, short box lengths reflect a tighter clustering of SNP copy number values than do longer box lengths. The midpoint of each box represents the median CNA value for all samples. An illustration figure was displayed in Figure 1.

2.6. Determination of Losses or Gains of Chromosome Arms. To determine loss or gain of a specific chromosome arm for a specific sample to produce Table 2 for the current study, we used a cutoff value of 10 on the cytoband summarized copy number data that were output from running the reb algorithm. A value larger than 10 was marked a gain for the 


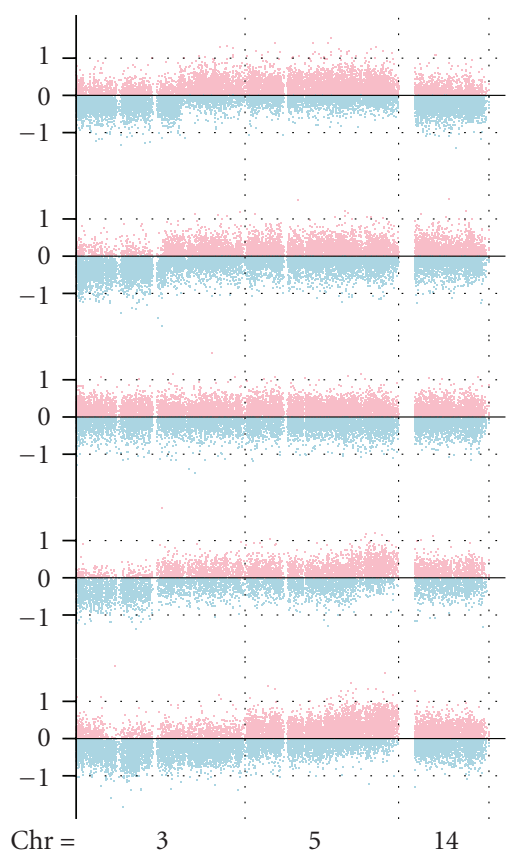

(a) rawCNs in $\log 2$ scale

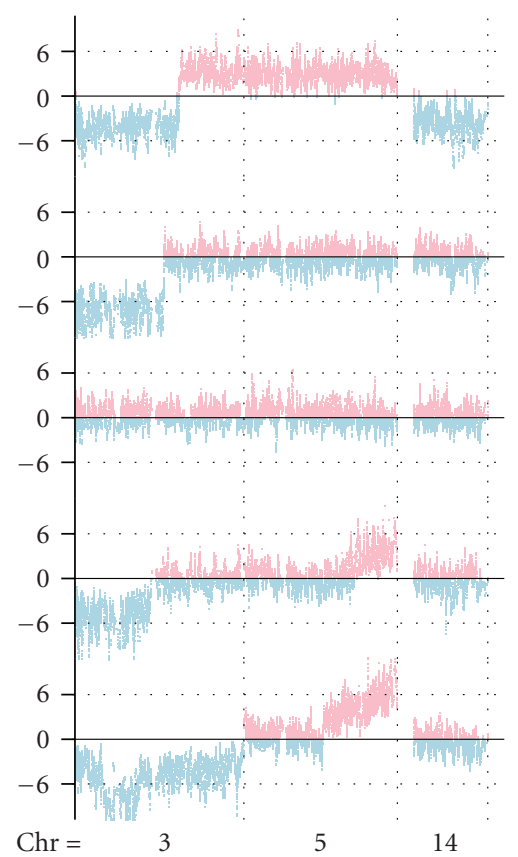

(b) Smoothed CNs

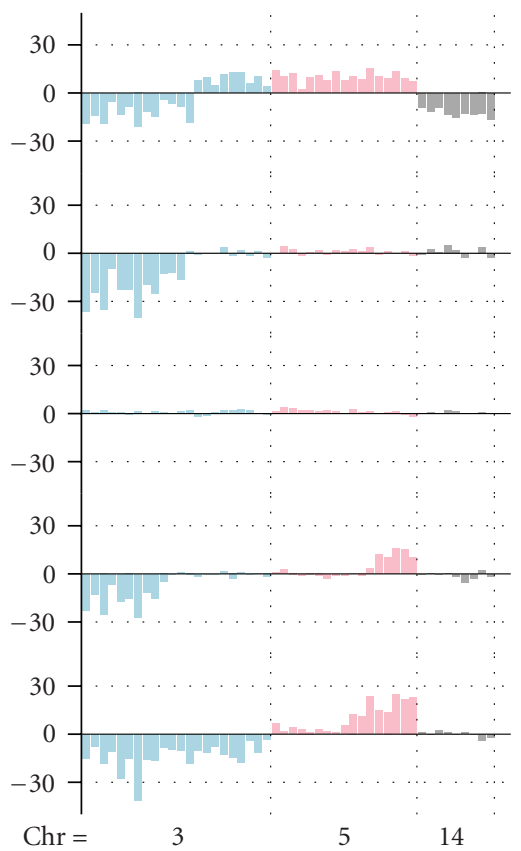

(c) Cyto summarized scores

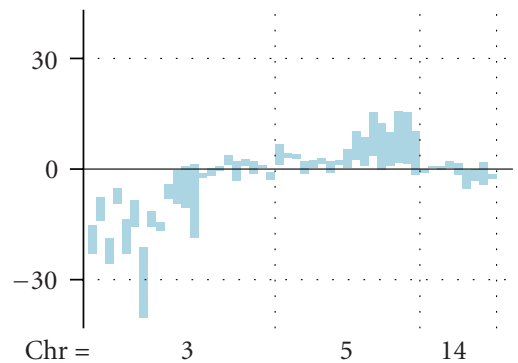

(d) Boxplot of scores

FIGURE 1: Illustration of algorithm for obtaining and displaying the cytoband summarized SNP copy number scores for a group of samples. (a) Display of raw SNP copy number alterations for individual tumor samples. Each horizontal line in the figure represents data from one tumor sample (total of five tumor samples displayed). Raw SNP copy numbers are displayed in log 2 scale and plotted on the $Y$-axis. Negative copy numbers (indicating SNP losses) are depicted in blue and positive copy numbers (indicating SNP gain) are depicted in pink. The $X$-axis represents the physical ordering of individual SNPs along different chromosomes. (b) Display of smoothed SNP copy number alterations for individual tumor samples. Each horizontal line represents data from one tumor sample (total of five tumor samples displayed). (b) the smoothed copy number by moving $t$-test with window size 31 , (c) the cytoband summarized SNP copy number scores using the adapted regional expression bias algorithm, and (d) boxplot of the summarized SNP copy number scores. 
whole cytoband for the sample, while a value less than -10 was marked a loss for the cytoband. The proportions of losses or gains for each cytoband across all tumors in the study group were calculated. The proportion of loss (gain) of an arm was determined to be the highest loss (gain) proportion among all cytobands within the arm. If the proportion of loss was higher than that of gain, the arm was an overall loss and vice versa (undecided cases when the loss proportion equals to the gain proportion are unlikely to happen for the selected arms). The numbers and proportions of losses or gains for selected chromosome arms calculated this way were then summarized in Table 2.

\section{Results}

3.1. RCC Tumor Subtypes have Distinct Cytogenetic Alteration Profiles. First, we compared the cytogenetic profiles of the four RCC subtypes: clear cell, papillary, chromophobe, and oncocytoma (Figures 2(a) and 2(b)). Unsupervised clustering of tumor samples based on their cytogenetic data revealed four clusters in the plot, roughly corresponding to the four RCC subtypes. Tumors of a given subtype generally clustered together. For example, all tumors of clear cell type clustered together, with the exception of one case which clustered with chromophobe samples. Our results indicate that each RCC subtype displays a distinct cytogenetic alteration profile (Figure 2(a)). This cytogenetic profiling correctly predicted tumor subtypes with an overall accuracy of $92 \%$ (65/71). Although we cannot be certain, we speculate that tumors misclassified by this analysis had mixed features or were misdiagnosed.

3.2. $-3 p,+5 q$, and $-8 p$ Are Unique Events for ccRCC. Comparing cytogenetic profiles of four RCC tumor subtypes, we found that $-3 p,+5 q$ and $-8 p$ are unique to ccRCC tumors (Figure 2(b)). Tumor subtypes were listed in increasing order of cytogenetic complexity, from having the least alterations (oncocytoma) to the most ones (chromophobe). Apart from oncocytoma, which is a benign tumor and does not possess any obvious cytogenetic changes, $+7,+12$, and -16 were seen to occur in all other RCC subtypes, but to varying degrees. This suggests there may be common cytogenetic alterations affecting shared signaling pathways in these RCC subtypes. Interestingly enough, we observed that there were no regions having copy number losses which were common to all three malignant RCC tumors. $-14 \mathrm{q}$ was seen in ccRCC and papillary RCC only, while $+14 \mathrm{q}$ was seen in chromophobe RCC. -3 p was seen in ccRCC tumors only, while $+3 p$ was seen in papillary RCC tumors and $3 p$ was unchanged in chromophobe RCC. Therefore, it is likely that each RCC subtypes has a distinct tumor initiation (such as gene mutations) patterns, especially for TSGs if they are involved in the development of RCCs. As the VHL gene is located on 3p25-26, this indicates that deregulation of VHL pathway is involved for ccRCC development only, but not for the other tumor subtypes. Thus, different RCC subtypes have unique cytogenetic alterations as well as common alterations. As ccRCC accounts for the majority of RCC tumors, we will focus our analysis on this subtype only in the rest of paper.
3.3. $-3 p,+5 q,+7$, and $-14 q$ Are Associated with Early Tumor Stages of $c c R C C$. Tumor stage is an important factor in determining tumor progression. We grouped ccRCC tumors and analyzed their cytogenetic alteration profiling according to tumor stages (Figure 3(a)). As was expected, we saw that cytogenetic alteration events increased as tumor stage increased. Tumors at the earliest stage, S1a, had the least cytogenetic alterations with $-3 p,+5 q,+7$, and -14 only, while tumors at the latest stages, S3b + S4 (the only case of stage 4 tumor was merged with $\mathrm{S} 3 \mathrm{~b}$ ) had the most cytogenetic alterations, notably $-1 \mathrm{p},+1 \mathrm{q},-3 \mathrm{p},+5 \mathrm{q},-6 \mathrm{q},+7,-8 \mathrm{p}$, $+8 \mathrm{q},-9,+12,-13,-14,-18,+20$, and -22 . This agrees with the prevailing model of tumorigenesis and progression: as tumor progresses, initial simple genetic events (sequence mutations, segmental losses or gains) trigger more and more cytogenetic alterations through altering the tumor cell microenvironment, causing the tumor genome to become less stable. Loss in chromosome 17 has been previously reported to be associated with later stage events of RCC [23]; however, in our samples, we did not find obvious loss or gain of whole and/or part of chromosome 17.

3.4. $-3 p$ and $+5 q$ Are the Most Frequent Events for ccRCC Tumors and Are Weakly Related. Our data suggest that $-3 \mathrm{p}$ and $+5 q$ are universal events in all stages of ccRCC tumors (Figure 3(a)) and in all grades of tumors (Figure 3(b)). In other word, they appeared to be unrelated to tumor stage and grade. In later stages, losses in $3 \mathrm{p}$ were largely unchanged in frequencies (around 70\%-80\%, Table 2), but gains in $5 \mathrm{q}$ occurred with varied frequencies and degrees (Table 2 and Figure 3(a)). The two events appeared to be weakly inversely related to each other (Pearson's correlation test with correlation coefficient $=-0.25, P=.12)$. As unbalanced translocations between $3 p$ and $5 q$ were frequently observed [24], this result implies the possibility of $5 q$ gain without $3 p$ loss or vice versa. Indeed, Podolski and colleagues studied $5 \mathrm{q}$ gains intensely, found that there was a high frequency in an unbalanced translocation between $3 p$ and $5 q$, leading to the $3 p$ loss of one of its parental copies and $5 q$ gains. $5 q$ gain could also occur in independent ways to $3 p$ loss, such as to have unbalanced translocations with other chromosomes [25]. The most frequent losses on $3 p$ were $3 \mathrm{p} 26,3 \mathrm{p} 24$, 3 p14 and 3p21-22 for the early-stage tumors (Figure 3(a) and Supplementary Figure S1A in supplementary material available online at doi:10.1155/2010/428325, detailed plot of $3 p$ is not shown). $+5 q$ occurred most frequently for stage 1a tumors at 5q31-5qter. It reached its peak at intermediate stages (S1b and S2), then started to decrease at later stages of S3 and S4. This observation gives partial support of the previously reported result that $5 \mathrm{q}$ gain was associated with good prognosis of ccRCC patients [24, 25]. We will discuss this in more detail in the following section.

3.5. $5 q$ Gain Is Likely Involved in the Transition from Stage $1 a$ to Stage $1 b$ Tumors and May Play a Critical Role in ccRCC Development. The profiles of cytogenetic alterations between stage $1 \mathrm{a}$ and stage $1 \mathrm{~b}$ tumors were remarkably similar (See Figure 3(a) and Supplementary Figures S1A, 


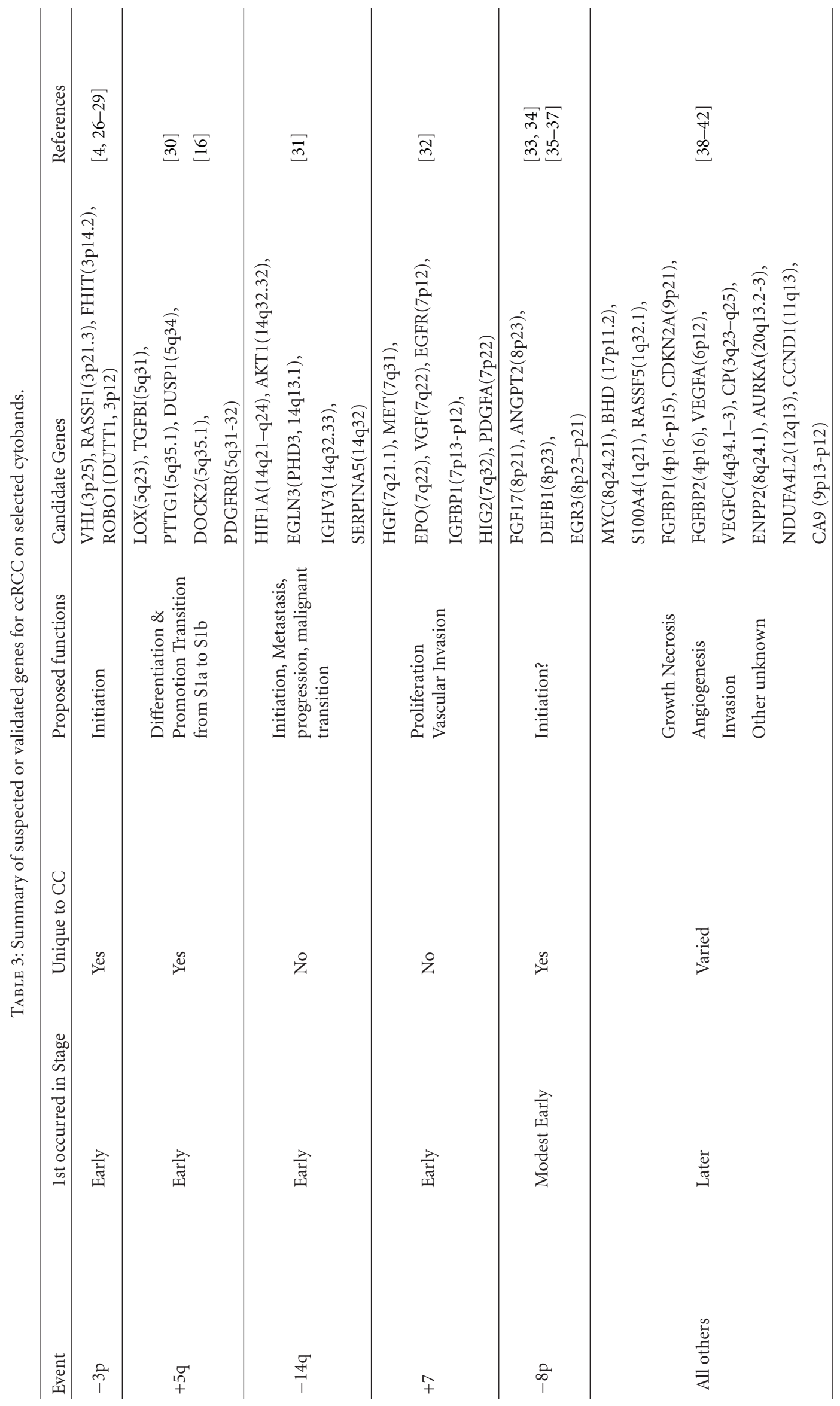




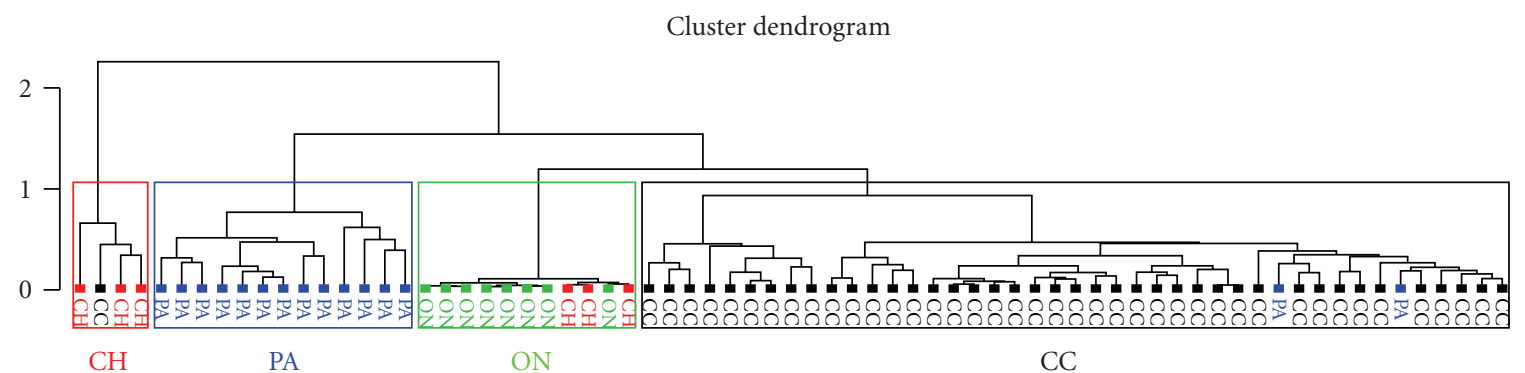

(a)

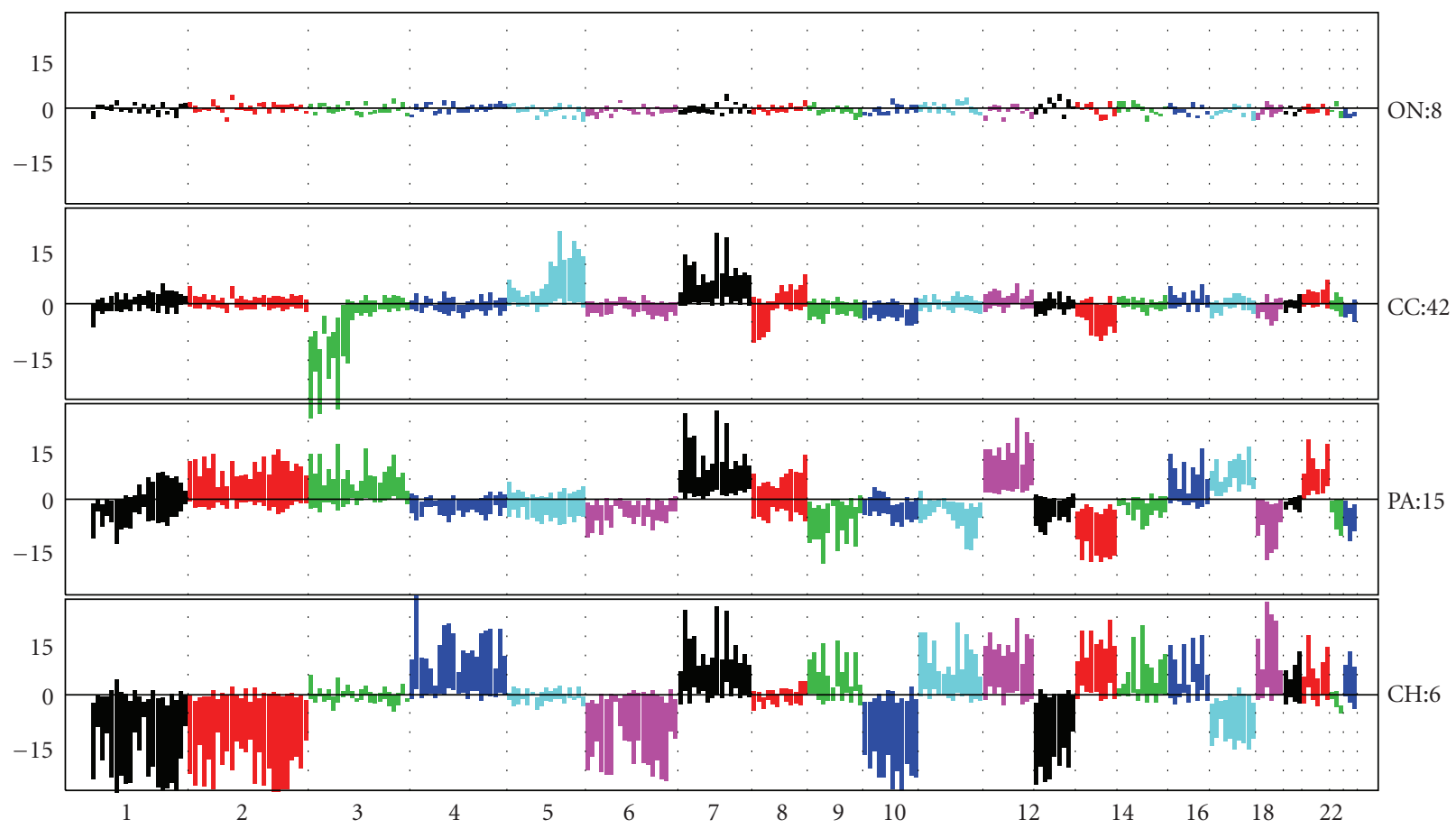

(b)

FIGURE 2: (a) Unsupervised clustering of RCC samples based on cytoband summarized SNP data. CH: chromophobe RCC, PA: papillary RCC; ON: oncocytoma, CC: clear cell. With few exceptions, tumors of a given subclass clustered together. Thus, each of the four RCC subtypes has a distinct pattern of cytogenetic alterations. (b) Somatic cytogenetic alteration profiles of RCC subtypes. RCC subtypes are ordered by complexity of molecular cytogenetic alterations. Each bar in the plot is a box with upper and lower bound of the box representing the third (75\%, upper bound) and first quantiles (25\%, lower bound) over the summarized SNP copy numbers by cgma method. The boxes are ordered from $\mathrm{p}$-arm to q-arm followed by another chromosome. Only the somatic chromosomes are shown here. A positive value stands for gain, while a negative value stands for loss. Each chromosome is assigned a single color different from its neighbor chromosomes.

S1B). A formal studentized $t$-test was used to test the difference of cytogenetic alterations between the two groups of tumors having stages $1 \mathrm{a}$ and $1 \mathrm{~b}$. A test statistic below -2 or above 2 was used to declare the differences to be significant. The profile of test $P$-values was plotted in Supplementary Figure S1C. The identified cytogenetic alterations declared to be significant between tumor groups $\mathrm{S} 1 \mathrm{a}$ and $\mathrm{S} 1 \mathrm{~b}$ were 3p (p25-p22), 5q (q34-q35), 4q23, 11q24 and 17q22. Apart from the single cytoband on chromosomes 4,11 , and 17 , the most significant differences were recognized as more gains in $5 q$ and more losses in $3 p$ in stage $1 b$ tumors than in stage 1a tumors. Unlike gains in chromosome $7,+5 q$ is seen to be unique to ccRCC tumors (Figure 2(b)); it occurs in a large proportion of ccRCC tumors (43\%, Table 3 ).
To figure out in more details, we then used a $3 \mathrm{~cm}$ and $7 \mathrm{~cm}$ as criteria for grouping the tumors into one of the small-sized $(<3 \mathrm{~cm})$, medium-sized and large-sized $(>7 \mathrm{~cm})$ groups. The cytogenetic alteration profile of the 9 smallsized tumors was compared with those of medium- or largesized tumors (Supplementary Figures 2D-2F. The profiles for the small and medium sized tumors were remarkably similar, with the only striking difference occurred in $5 \mathrm{q}$, where there were no obvious gains in the small sized tumors and where there were a significant more $5 \mathrm{q}$ gains in the medium sized tumors. Therefore, a critical role can be proposed for genes in this region during ccRCC formation, whose gene disruptions promote the tumor growth in size and differentiate the kidney tumor cells into well-defined 


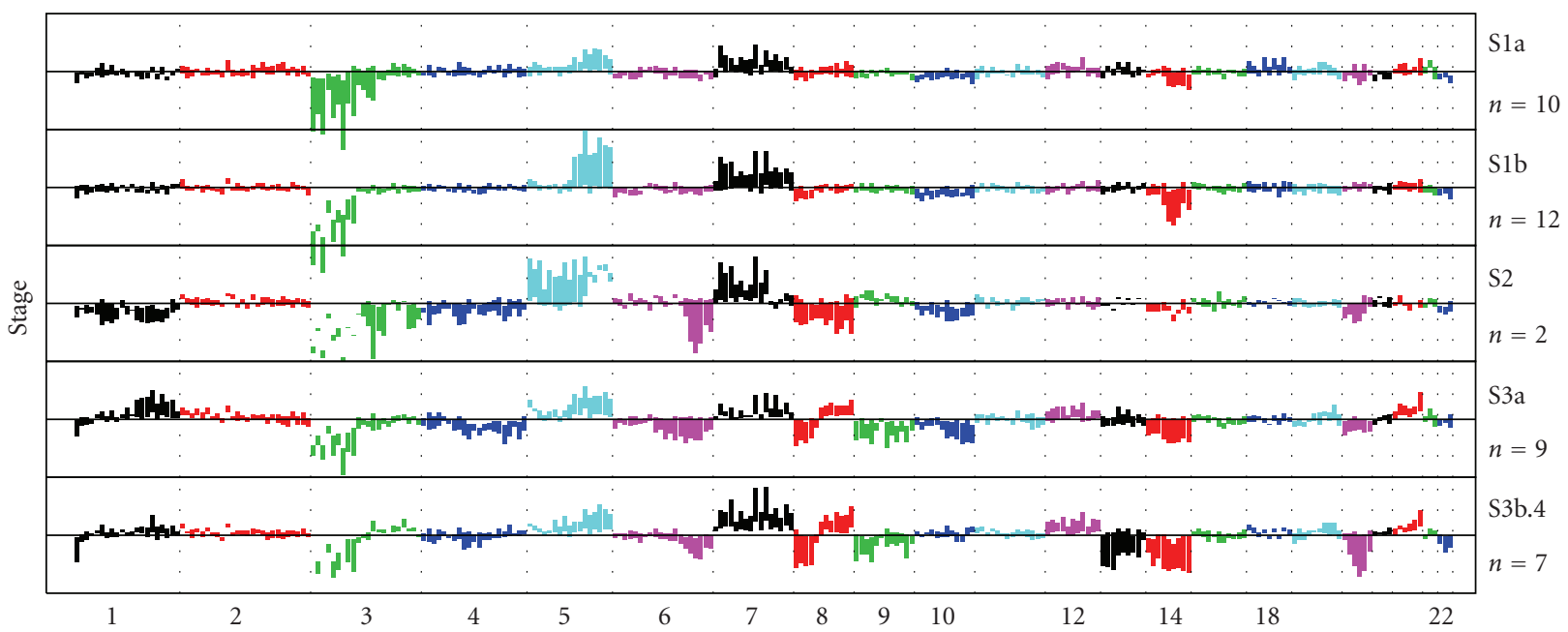

(a)

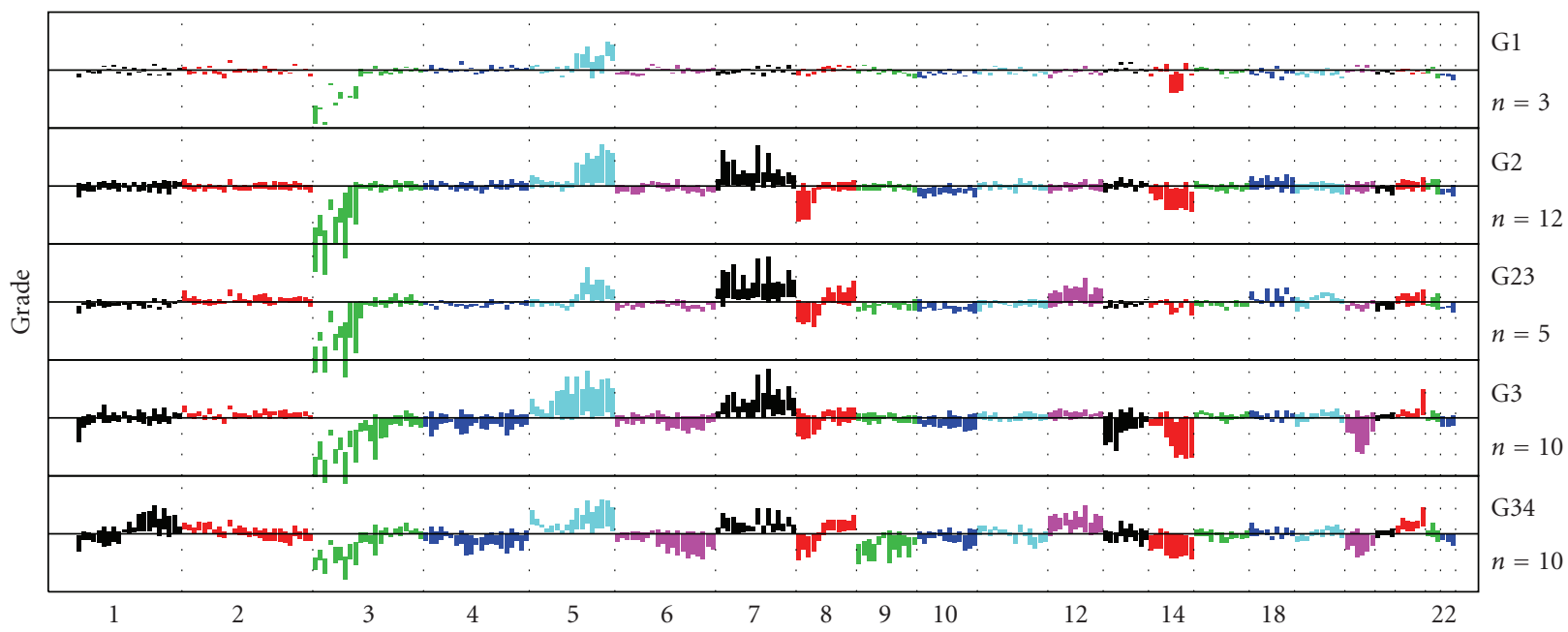

(b)

FIgURE 3: (a) Somatic cytogenetic alteration summarization based on SNP data, grouped by patient tumor stages in increasing order. Box plots were arranged as in Figure 2. The earliest stage S1a shows the fewest cytogenetic alterations, while the highest stage S3b4 shows the largest number of cytogenetic changes, indicating the cytogenetic progression of ccRCC tumors. $-3 p,+5 q,+7,-8 p$, and -14 are among the earliest events during ccRCC tumorigenesis, while $-1 \mathrm{p},+1 \mathrm{q},-4 \mathrm{p},-6 \mathrm{q},-9 \mathrm{p},+12,-13,-18$, and $+20 \mathrm{q}$ are events occurring at later stages only. (b) Somatic cytogenetic alterations associated with tumor grade. Box plots are arranged as in Figure 2.

clear cell tumors. Cancer-relevant genes on $5 \mathrm{q}$ include the LOX gene, which has been shown to play an essential role in hypoxia-induced metastasis [30], the later is triggered by the disrupted VHL gene in the tumor cells. Other genes in the area include $P D G F R B$, which plays an important role in tumor neovascularization [43], TGFBI [16], PTTG1, DOCK2 and DUSP1 (the latter three genes were speculated based on our internal studies), which were likely to be involved in ccRCC progression and development. However, no mutation genes were found in this region so far.

\section{6. $14 q$ Likely Contains Important TSG Genes Unrelated to} $3 p$ Loss. As we see from our data, $-14 \mathrm{q}$ occurred in the earliest stage and lowest grade tumors too (along with $-3 p$, Figures 3(a) and 2(b)); it displayed a clear increasing pattern in occurrence frequencies along tumor stages, uncorrelated with that of $-3 p$ occurrences (Table 3 ). This suggests that $-14 \mathrm{q}$ is likely to have occurred in an independent way to $3 p$ loss; it also indicates that its occurrences are also influenced by the tumor cell environment: as later and more advanced tumors tend to have more occurrences of $-14 \mathrm{q}$. Tumor initiation and promotion roles in ccRCC tumor development can be proposed for genes in this region. Disruptions of one or more than one key TSG genes in this region were likely involved during the early ccRCC tumorigenesis. At the same time, some functionally important genes may also be activated by other events of tumors; those genes may play a significant role in promoting tumor invasiveness, as was shown in Supplementary Figure S2I and S2J. 14q contains functionally diversified genes, critical to a few important and 
well-studied pathways, such as HIF1 $\alpha$ (14q21-q24, [44]) and EGLN3(PHD3, 14q13.1, [45]), both of which were shown to be disrupted in ccRCC tumors, due to the disrupted VHL gene functions. The heavy chains of human antibodies (or immunoglobulins) are also located in the far end of $14 \mathrm{q}$, with often elevated activities in ccRCC tumors. On the other hand, other known involved tumor suppressor genes in this region were AKT1 (14q32.32, [46]) and SERPINA5 (14q32, [31]). AKT1 gene was shown to play a pivotal role in the AKT/PI3K signaling pathway. In summary, our data suggest that genes in $14 \mathrm{q}$ play important and diversified roles in both ccRCC formation and its development. Again, much remains unknown about the roles the genes in this region play during ccRCC formation and development. More study of genes in the region is needed to better understand the ccRCC tumorigenesis and progression.

3.7. Gain of Whole or Part of Chromosome 7 Is an Early Stage Event of ccRCC, Independent of Tumor stage. Gain of chromosome 7 was seen to appear in all stages (Figure 3(a)), indicating that one or more genes on 7 may play an important role in both tumor development and tumor growth. Gain of chromosome 7 also occurred in tumors of all grades except grade 1 (Figure 3(b)). The most active cytoband was identified to be 7q21-7q31 (data not shown). These regions contain a number of functionally important genes, including MET (also being called HGFR, RCCP2, c-Met) in 7q31, HGF in $7 \mathrm{q} 21, E P O$ (Human erythropoietin gene) in $7 \mathrm{q} 22, V G F$ (nerve growth factor inducible gene) in $7 q 31$ and PDGFA (platelet-derived growth factor alpha) in 7 p22 or IGFBP1 (7p13-p12). Amplification of or activating mutations in these genes may play a role in the development of ccRCC. Unlike $+5 \mathrm{q},+7$ was not unique to ccRCC, but that it occurs in all malignant RCCs; a general role for genes in this region can be suggested during RCC development, such as proliferation and vascular invasion (Supplementary Figure S2J), leading to poor patients' survivals (Supplementary Figure S2A).

3.8. -1 pter, $+1 q,-4-6 q,+8 q,-9,+12,-13,-18$, and +20 Are Later Stage Events in ccRCC. These events did not appear in the early stage S1a or S1b tumors, but occurred progressively as tumors advance in stage. For example, -13 and -18 occurred only when tumor stages exceeded 2 , and they peaked at the latest stage (S3b4, Figure 3(a)). These events also occurred in high-grade tumors only (see Figure 3(b)). This pattern suggests that these cytogenetic alterations are generally the consequences of tumor development due to increased genomic material instability, rather than the cause of it. However, these genetic alterations may still play critical roles in tumor progression, metastasis, and/or proliferation. In a recent study [14], the authors found that cytogenetic alterations of $-4 / 4 \mathrm{p}$ and $-9 / 9 \mathrm{p}$, among other alterations, were significantly associated with patients' survivals. We will discuss more about these regions in the coming sections.

3.9. Association between Cytogenetic Alterations in ccRCC and Tumor Grade. Next, we grouped and analyzed cytogenetic alterations in ccRCC by tumor grade (Figure $3(\mathrm{~b})$ ). The patterns were very similar to that grouped by tumor stage. $-3 p,+5 q$, and $-14 q$ were seen to appear in all grades of ccRCC tumors. On the other hand, $-1 \mathrm{p} 36,-4,-6,+12$, $-13,-18$ and +20 were seen in high-grade tumors only. Of the three tumors with grade 1 , we did not see obvious +7 or $-8 \mathrm{p}$. $-8 \mathrm{p}$ appeared to occur after $-3 \mathrm{p},-14 \mathrm{q}$, and $+5 \mathrm{q}$. Due to the small size of grade 1 tumors ( 3 cases), the clear display of $-8 \mathrm{p}$ in grade 2 tumors (12 cases), and relative high frequency of occurrence in clear cell tumors $(24 \%$, see Table 3 ), we decide that $-8 \mathrm{p}$ is a moderate early event in the process of ccRCC tumorigenesis. We noted subtle differences between the patterns of cytogenetic alterations associated with tumor grade compared to alteration patterns with tumor stage. +7 was seen in stage la tumors, yet it was not seen in grade 1 tumors. This discrepancy may reflect differences in the clinical features of tumor grade and stage.

3.10. Association of Cytogenetic Alterations in ccRCC with Patient Survivals and Other Clinical or Pathological Factors. Next, we examined cytogenetic alterations in ccRCC tumors in association with patient survivals. The averaged significant $P$-values for each cytoband were plotted in Supplementary Figure S2A. Cytobands from chromosomes 1, 2, 4, 6, 7, 8 , and 13 were identified to be associated with patients' survivals. Specific cytogenetic bands associated with patient survival were summarized in Supplementary Table 1. Among these regions, $-4 \mathrm{p} / 4$ and -13 were seen to be most significantly associated with poor survivals. This was confirmed in previous study [14]. +1q was seen to be correlated with quite a few clinicopathological factors, which include sarcomatoid element, stage, vein, and vascular invasions. It is no surprise that $+1 \mathrm{q}$ is associated with patients' survivals (Supplementary Figure S2). Other regions were also identified to have significant prognostic significance, which were summarized in Supplementary Table 1. Due to our limited number of available cases who have follow-up data, the results displayed here are likely to be conservative.

Associations between cytogenetic alterations in ccRCC and other clinicopathological features, including VHL mutation status, gender, tumor size, sarcomatoid elements, gross tumor necrosis, renal vein and vascular invasions were examined too; the significances of tests were summarized in Supplementary Table 2 and displayed in Supplementary Figure S2. Of the different profiles associated with different clinical or pathological factors, the profiles for tumor grade (Grade 1-2 versus Grade 3-4, Figure S2D), for tumor stage (Stage 1-2 versus Stage 3-4, Figure S2E) and for tumor size (size < versus $\geq 4 \mathrm{~cm}$, Figure S2F) are comparably similar, while the profiles for vein invasion (Yes versus No, Figure S2I) and for vascular invasion (Yes versus No, Figure S2J) are remarkably similar. These similarities reflect the closeness of being clinicopathological factors. We note that, interestingly, VHL mutation status is not associated with any specific cytogenetic changes and that $+1 \mathrm{q}$ is associated with sarcomatoid differentiation, tumor vein and vascular invasions as well as poor patient survivals, indicating that some genes in this gene rich area play critical roles in tumor progression or metastasis. The cytogenetic 


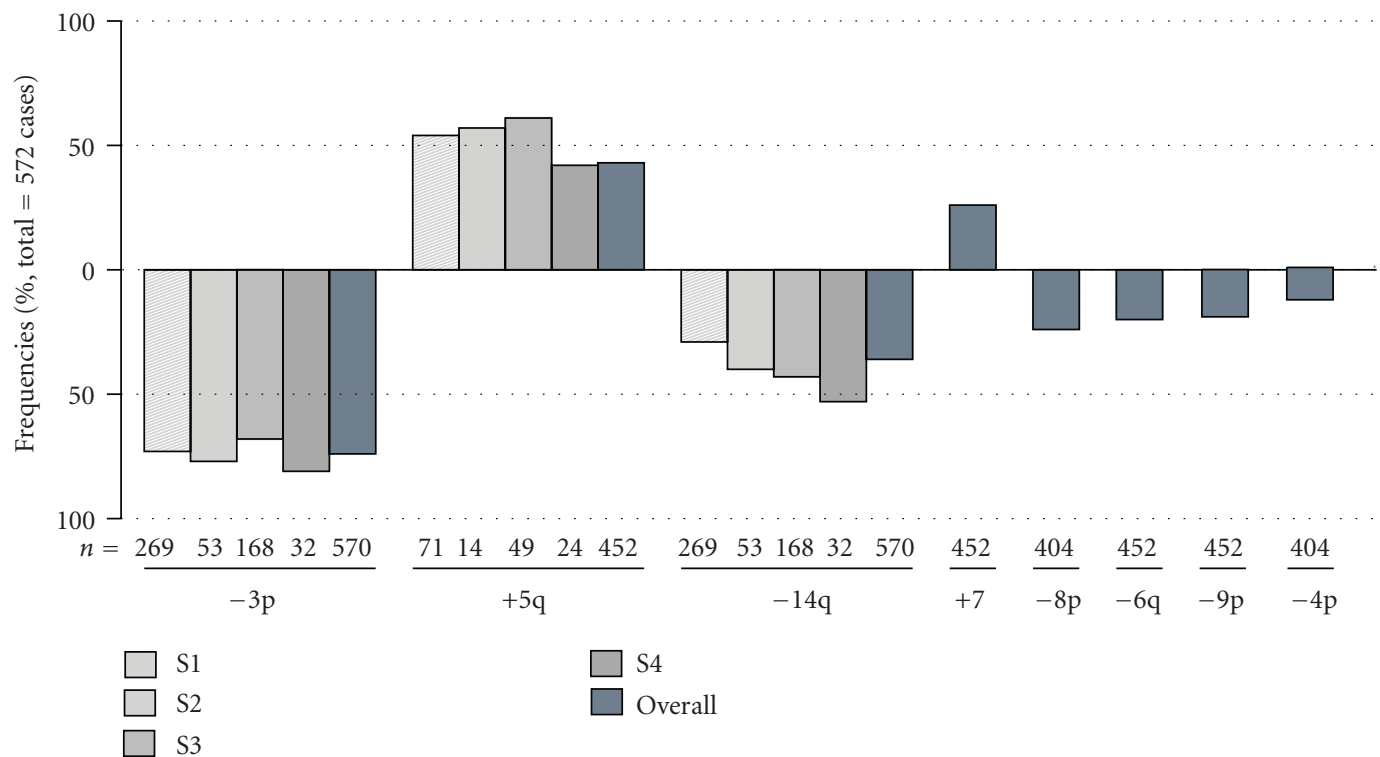

FIGURE 4: Relative frequencies of cytogenetic alterations in 572 ccRCC tumors from multiple sources.

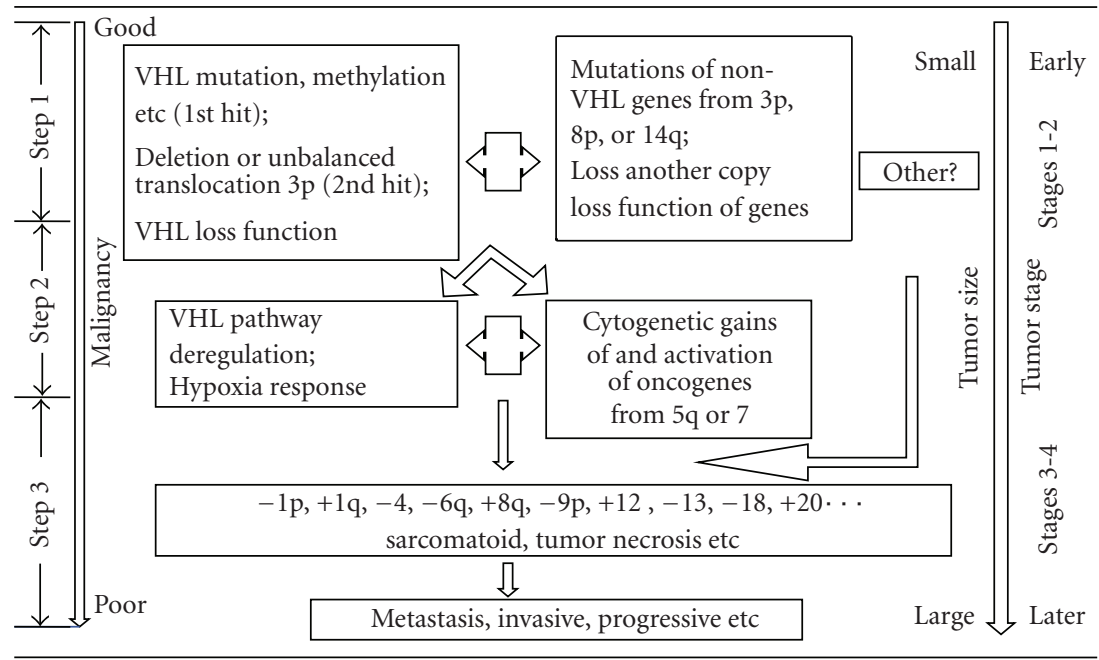

FIGURE 5: Illustration of a proposed model of ccRCC tumor formation and progression.

profiles between male and female patients were remarkably similar too (Figure S2C), except that male patients were more likely to have amplified chromosome 7 and $1 \mathrm{q}$ than their female counterparts, and that female patients were more likely to exhibit $8 p$ loss. Further studies were needed to verify if these differences were gender related.

\section{Discussion}

We have studied the whole genome cytogenetic alterations in RCC tumors using high-density high-throughput Affymetrix SNP arrays. We confirmed that different subtypes of RCC had distinct cytogenetic profiles, reflecting the heterogeneity of RCC. We also showed that specific cytogenetic alterations in ccRCC are associated with specific clinicopathological features. Finally, by examining cytogenetic profiles from ccRCC tumors at different stages of progression, we will be able to construct a detailed map of the sequential cytogenetic changes that occur during ccRCC progression below.

Based on results in Table 2 and Figure 4 of the combined studies, the most frequent alterations for ccRCC were identified as $-3 \mathrm{p}(74 \%),+5 \mathrm{q}(43 \%),-14 \mathrm{q}(36 \%),+7(26 \%)$, and $-8 \mathrm{p}(24 \%)$. In the four studies where tumor stage information was available, $-3 p$ seemed to display no patterns of decrease or increase in frequency as tumor stage increases, while $-14 \mathrm{q}$ occurrences show an increasing pattern in frequency along tumor stages. In all studies, $-14 \mathrm{q}$ occurred in at least $20 \%$ of stage 1 tumors (average: 29\%), then increased in occurrence frequencies as tumor stage increases. This indicates that $-14 \mathrm{q}$ is among the earliest events in 
the tumorigenesis process. In our study, $-8 \mathrm{p}$ occurred in ccRCC tumors only and occurred in almost all grade and stage tumors. This comparison indicates that $-8 \mathrm{p}$ may have occurred in early but not earlier than $-3 p$ and $-14 q$ did.

First of all, based on our experience and mouse model study as well as published papers [47], we postulate that there exist more than one independent pathway for ccRCC tumors to form. The majority of ccRCC tumors involve loss of function of the VHL gene, a key regulator of the hypoxia-response pathway. Loss of function of VHL leads to unregulated activity of HIF, a hypoxia-inducible transcription factor. Overactivity of HIF, in turn, leads to uncontrolled activation of the hypoxia-response pathway. The VHL gene has been reported as mutated or methylated in over $70 \%$ of ccRCC tumors [48]. However, studies in our laboratory (data not shown) and that in others indicate that more than $90 \%$ of ccRCC tumors exhibit deregulation of the VHL pathway. This suggests an independent and parallel event(s) to VHL gene mutation for deactivation of the VHL functional pathway. Deactivation of VHL pathway alone is not sufficient to cause the ccRCC phenotype $[49,50]$.

Combining all the information, we have collected, we propose a new model of ccRCC tumorigenesis and progression. This model is illustrated in Figure 5. For the majority of ccRCC tumors where VHL is involved, the first step starts when a key event (mutations, methylation, etc.) occurs in VHL, leading to deactivation of the VHL pathway and unregulated hypoxia response. Thereafter, the tumor cells are constantly under altered microenvironment, paving the way toward tumorigenesis. If later on, some key non-VHL TSG genes from 3p, 8p, and/or 14q undergo mutations or gene alterations, leading to the losses of the functions of the corresponding genes, the tumor cells then get sufficient potential toward tumorigenesis, finishing the first step of tumor formation in the sequence. In the second step, key proto-oncogenic genes from $5 \mathrm{q}$ or 7 are activated due to either genetic or nongenetic reasons, such as gain-of-function mutations, gene regulations, or cytogenetic gains or the sustained microenvironment alterations around the tumor cells. Proto-oncogene mutation here is not a necessary condition. The tumor cells at this step will transit from previous latent state to present, from being local to more opt to proliferate and metastasize; the general results include a sudden increase in tumor size and further destabilized microenvironment, triggering more genetic and cytogenetic denormalization. Incidentally, deactivation of the VHL pathway can itself lead to heightened activity of VEGF and PDGF. As further genetic and cytogenetic events accumulate and more signaling pathways are deregulated, the tumor moves into the third step of progression, eventually becoming invasive and metastatic. We believe that this model describes the genetic progression of the majority of ccRCC tumors, although VHL-pathway-independent mechanism of the tumorigenesis is not excluded for a minority of tumors (Figure 5, right-hand side). Candidate tumor suppressor genes and/or oncogenes in this process were summarized in Table 3, based on the literature and our experiences. Some of these genes were well studied. Examples are the RASSF1 gene on 3p21.3 [26], FHIT on 3p14.2 [51], BHD gene on
$17 \mathrm{p} 11.2[38,39]$, and AKT1 gene on $14 \mathrm{q} 32$ [40]. The rest of genes were selected based on either literature or results of our internal gene expression profiling.

\section{Acknowledgments}

The authors are thankful to the cancer centers of Spectrum Health Hospital in Grand Rapids and the Cooperative Human Tissue Networks (CHTN) for allowing them to use their tissue samples for this microarray study. They are also in debt to their technical editor Vanessa Fogg for her great help in preparation of this manuscript and Dr. Bin Teh and Dr. Kyle Furge for their generous support for this research.

\section{References}

[1] A. G. Knudson Jr., "Mutation and cancer: statistical study of retinoblastoma," Proceedings of the National Academy of Sciences of the United States of America, vol. 68, no. 4, pp. 820823, 1971.

[2] M.-H. Tan, C. G. Rogers, J. T. Cooper et al., "Gene expression profiling of renal cell carcinoma," Clinical Cancer Research, vol. 10, no. 18, part 2, pp. 6315S-6321S, 2004.

[3] H. T. Cohen and F. J. McGovern, "Renal-cell carcinoma," The New England Journal of Medicine, vol. 353, no. 23, pp. 24772490, 2005.

[4] F. Latif, K. Tory, J. Gnarra et al., "Identification of the von Hippel-Lindau disease tumor suppressor gene," The Science, vol. 260, no. 5112, pp. 1317-1320, 1993.

[5] B. F. Skinnider, A. L. Folpe, R. A. Hennigar et al., "Distribution of cytokeratins and vimentin in adult renal neoplasms and normal renal tissue: potential utility of a cytokeratin antibody panel in the differential diagnosis of renal tumors," American Journal of Surgical Pathology, vol. 29, no. 6, pp. 747-754, 2005.

[6] M. Takahashi, X. J. Yang, J. Sugimura et al., "Molecular subclassification of kidney tumors and the discovery of new diagnostic markers," Oncogene, vol. 22, no. 43, pp. 6810-6818, 2003.

[7] N. H. Cho, S. Kim, M. J. Ha, and H. J. Kim, "Simultaneous heterogenotypic renal cell carcinoma: immunohistochemical and karyoptic analysis by comparative genomic hybridization," Urologia Internationalis, vol. 72, no. 4, pp. 344-348, 2004.

[8] K. Junker, G. Weirich, M. B. Amin, P. Moravek, W. Hindermann, and J. Schubert, "Genetic subtyping of renal cell carcinoma by comparative genomic hybridization," Recent Results in Cancer Research, vol. 162, pp. 169-175, 2003.

[9] J. P. Kallio, E. H. Mahlamäki, H. Helin, R. Karhu, P. Kellokumpu-Lehtinen, and T. L. J. Tammela, "Chromosomal gains and losses detected by comparative genomic hybridization and proliferation activity in renal cell carcinoma," Scandinavian Journal of Urology and Nephrology, vol. 38, no. 3, pp. 225-230, 2004.

[10] D. Reutzel, M. Mende, S. Naumann et al., "Genomic imbalances in 61 renal cancers from the proximal tubulus detected by comparative genomic hybridization," Cytogenetics and Cell Genetics, vol. 93, no. 3-4, pp. 221-227, 2001.

[11] M. A. Rigola, C. Casadevall, M. Bernués et al., "Analysis of kidney tumors by comparative genomic hybridization and conventional cytogenetics," Cancer Genetics and Cytogenetics, vol. 137, no. 1, pp. 49-53, 2002. 
[12] J. Sanjmyatav, J. Schubert, and K. Junker, "Comparative study of renal cell carcinoma by CGH, multicolor-FISH and conventional cytogenic banding analysis," Oncology Reports, vol. 14, no. 5, pp. 1183-1187, 2005.

[13] C. Béroud, J.-C. Fournet, C. Jeanpierre et al., "Correlations of allelic imbalance of chromosome 14 with adverse prognostic parameters in 148 renal cell carcinomas," Genes Chromosomes and Cancer, vol. 17, no. 4, pp. 215-224, 1996.

[14] T. Klatte, P. N. Rao, M. De Martino et al., "Cytogenetic profile predicts prognosis of patients with clear cell renal cell carcinoma," Journal of Clinical Oncology, vol. 27, no. 5, pp. 746-753, 2009.

[15] B. Gunawan, W. Huber, M. Holtrup et al., "Prognostic impacts of cytogenetic findings in clear cell renal cell carcinoma: gain of 5q31-qter predicts a distinct clinical phenotype with favorable prognosis," Cancer Research, vol. 61, no. 21, pp. 7731-7738, 2001.

[16] D. Matsuda, S. K. Khoo, A. Massie et al., "Identification of copy number alterations and its association with pathological features in clear cell and papillary RCC," Cancer Letters, vol. 272, no. 2, pp. 260-267, 2008.

[17] R. Beroukhim, J.-P. Brunet, A. Di Napoli et al., "Patterns of gene expression and copy-number alterations in von-Hippel Lindau disease-associated and sporadic clear cell carcinoma of the kidney," Cancer Research, vol. 69, no. 11, pp. 4674-4681, 2009.

[18] F. Jiang, R. Desper, C. H. Papadimitriou et al., "Construction of evolutionary tree models for renal cell carcinoma from comparative genomic hybridization data," Cancer Research, vol. 60, no. 22, pp. 6503-6509, 2000.

[19] M. I. Toma, M. Grosser, A. Herr et al., "Loss of heterozygosity and copy number abnormality in clear cell renal cell carcinoma discovered by high-density affymetrix $10 \mathrm{~K}$ single nucleotide polymorphism mapping array," Neoplasia, vol. 10, no. 7, pp. 634-642, 2008.

[20] T. Yoshimoto, K. Matsuura, S. Karnan et al., "High-resolution analysis of DNA copy number alterations and gene expression in renal clear cell carcinoma," Journal of Pathology, vol. 213, no. 4, pp. 392-401, 2007.

[21] Y. Nannya, M. Sanada, K. Nakazaki et al., "A robust algorithm for copy number detection using high-density oligonucleotide single nucleotide polymorphism genotyping arrays," Cancer Research, vol. 65, no. 14, pp. 6071-6079, 2005.

[22] K. A. Furge, K. J. Dykema, C. Ho, and X. Chen, "Comparison of array-based comparative genomic hybridization with gene expression-based regional expression biases to identify genetic abnormalities in hepatocellular carcinoma," BMC Genomics, vol. 6, no. 1, article 67, 2005.

[23] J. C. Presti Jr., V. E. Reuter, C. Cordon-Cardo, M. Mazumdar, W. R. Fair, and S. C. Jhanwar, "Allelic deletions in renal tumors: histopathological correlations," Cancer Research, vol. 53, no. 23, pp. 5780-5783, 1993.

[24] K. Nagao, S. Yamaguchi, H. Matsuyama et al., "Allelic loss of 3 p25 associated with alterations of 5q22.3 q23.2 may affect the prognosis of conventional renal cell carcinoma," Cancer Genetics and Cytogenetics, vol. 160, no. 1, pp. 43-48, 2005.

[25] J. Podolski, T. Huzarski, T. Byrski et al., "Abnormalities of chromosome $5 \mathrm{q}$ correlate with morphologic features of better prognosis in clear cell renal carcinomas," Polish Journal of Pathology, vol. 46, no. 3, pp. 163-166, 1995.
[26] K. Dreijerink, E. Braga, I. Kuzmin et al., "The candidate tumor suppressor gene, RASSF1A, from human chromosome 3 p21.3 is involved in kidney tumorigenesis," Proceedings of the National Academy of Sciences of the United States of America, vol. 98, no. 13, pp. 7504-7509, 2001.

[27] I. Kuzmin, L. Liu, R. Dammann et al., "Inactivation of RAS association domain family $1 \mathrm{~A}$ gene in cervical carcinomas and the role of human papillomavirus infection," Cancer Research, vol. 63, no. 8, pp. 1888-1893, 2003.

[28] M. Velickovic, B. Delahunt, S. Störkel, and S. K. G. Grebe, "VHL and FHIT locus loss of heterozygosity is common in all renal cancer morphotypes but differs in pattern and prognostic significance," Cancer Research, vol. 61, no. 12, pp. 4815-4819, 2001.

[29] K. Hannula-Jouppi, N. Kaminen-Ahola, M. Taipale et al., "The axon guidance receptor gene ROBO1 is a candidate gene for developmental dyslexia," PLoS Genetics, vol. 1, no. 4, article e50, 2005.

[30] J. T. Erler, K. L. Bennewith, M. Nicolau et al., "Lysyl oxidase is essential for hypoxia-induced metastasis," Nature, vol. 440, no. 7088, pp. 1222-1226, 2006.

[31] T. Wakita, T. Hayashi, J. Nishioka et al., "Regulation of carcinoma cell invasion by protein $\mathrm{C}$ inhibitor whose expression is decreased in renal cell carcinoma," International Journal of Cancer, vol. 108, no. 4, pp. 516-523, 2004.

[32] Y. Miyata, S. Ashida, T. Nakamura et al., "Overexpression of hepatocyte growth factor receptor in renal carcinoma cells indirectly stimulates tumor growth in vivo," Biochemical and Biophysical Research Communications, vol. 302, no. 4, pp. 892897, 2003.

[33] Y.-T. Chen, J. J. Tu, J. Kao, X. K. Zhou, and M. Mazumdar, "Messenger RNA expression ratios among four genes predict subtypes of renal cell carcinoma and distinguish oncocytoma from carcinoma," Clinical Cancer Research, vol. 11, no. 18, pp. 6558-6566, 2005.

[34] A. N. Young, P. G. D. O. Salles, S. D. Lim et al., "Beta defensin-1, parvalbumin, and vimentin: a panel of diagnostic immunohistochemical markers for renal tumors derived from gene expression profiling studies using cDNA microarrays," American Journal of Surgical Pathology, vol. 27, no. 2, pp. 199205, 2003.

[35] M. M. Baldewijns, V. L. Thijssen, G. G. Van Den Eynden et al., "High-grade clear cell renal cell carcinoma has a higher angiogenic activity than low-grade renal cell carcinoma based on histomorphological quantification and qRT-PCR mRNA expression profile," British Journal of Cancer, vol. 96, no. 12, pp. 1888-1895, 2007.

[36] M. Yamakawa, L. X. Liu, A. J. Belanger et al., "Expression of angiopoietins in renal epithelial and clear cell carcinoma cells: regulation by hypoxia and participation in angiogenesis," American Journal of Physiology, vol. 287, no. 4, pp. F649-F657, 2004.

[37] M. J. Currie, S. P. Gunningham, K. Tumer et al., "Expression of the angiopoietins and their receptor Tie2 in human renal clear cell carcinomas; regulation by the von Hippel-Lindau gene and hypoxia," Journal of Pathology, vol. 198, no. 4, pp. 502-510, 2002.

[38] S. J. Mandriota, K. J. Turner, D. R. Davies et al., "HIF activation identifies early lesions in VHL kidneys: evidence for site-specific tumor suppressor function in the nephron," Cancer Cell, vol. 1, no. 5, pp. 459-468, 2002.

[39] M. L. Nickerson, M. B. Warren, J. R. Toro et al., "Mutations in a novel gene lead to kidney tumors, lung wall defects, 
and benign tumors of the hair follicle in patients with the Birt-Hogg-Dubé syndrome," Cancer Cell, vol. 2, no. 2, pp. 157-164, 2002.

[40] A. Kamb, N. A. Gruis, J. Weaver-Feldhaus et al., "A cell cycle regulator potentially involved in genesis of many tumor types," The Science, vol. 264, no. 5157, pp. 436-440, 1994.

[41] M. H. T. Bui, D. Seligson, K.-R. Han et al., "Carbonic anhydrase IX is an independent predictor of survival in advanced renal clear cell carcinoma: implications for prognosis and therapy," Clinical Cancer Research, vol. 9, no. 2, pp. 802-811, 2003.

[42] S.-W. Tang, W.-H. Chang, Y.-C. Su et al., "MYC pathway is activated in clear cell renal cell carcinoma and essential for proliferation of clear cell renal cell carcinoma cells," Cancer Letters, vol. 273, no. 1, pp. 35-43, 2009.

[43] J. I. Greenberg, D. J. Shields, S. G. Barillas et al., "A role for VEGF as a negative regulator of pericyte function and vessel maturation," Nature, vol. 456, no. 7223, pp. 809-813, 2008.

[44] J. D. Gordan, P. Lal, V. R. Dondeti et al., "HIF- $\alpha$ Effects on c-Myc distinguish two subtypes of sporadic VHL-deficient clear cell renal carcinoma," Cancer Cell, vol. 14, no. 6, pp. 435-446, 2008.

[45] E. Sato, T. Torigoe, Y. Hirohashi et al., "Identification of an immunogenic CTL epitope of HIFPH3 for immunotherapy of renal cell carcinoma," Clinical Cancer Research, vol. 14, no. 21, pp. 6916-6923, 2008.

[46] J. An and M. B. Rettig, "Mechanism of von Hippel-Lindau protein-mediated suppression of nuclear factor kappa B activity," Molecular and Cellular Biology, vol. 25, no. 17, pp. 7546-7556, 2005.

[47] S. C. Clifford, A. H. Prowse, N. A. Affara, C. H. C. M. Buys, and E. R. Maher, "Inactivation of the von HippelLindau (VHL) tumour suppressor gene and allelic losses at chromosome arm $3 p$ in primary renal cell carcinoma: evidence for a VHL-independent pathway in clear cell renal tumourigenesis," Genes Chromosomes and Cancer, vol. 22, no. 3, pp. 200-209, 1998.

[48] R. E. Banks, P. Tirukonda, C. Taylor et al., "Genetic and epigenetic analysis of von Hippel-Lindau (VHL) gene alterations and relationship with clinical variables in sporadic renal cancer," Cancer Research, vol. 66, no. 4, pp. 2000-2011, 2006.

[49] E. B. Rankin, J. E. Tomaszewski, and V. H. Haase, "Renal cyst development in mice with conditional inactivation of the von Hippel-Lindau tumor suppressor," Cancer Research, vol. 66, no. 5, pp. 2576-2583, 2006.

[50] W. Ma, L. Tessarollo, S.-B. Hong et al., "Hepatic vascular tumors, angiectasis in multiple organs, and impaired spermatogenesis in mice with conditional inactivation of the VHL gene," Cancer Research, vol. 63, no. 17, pp. 5320-5328, 2003.

[51] E. Braga, V. Senchenko, I. Bazov et al., "Critical tumorsuppressor gene regions on chromosome $3 p$ in major human epithelial malignancies: allelotyping and quantitative realtime PCR," International Journal of Cancer, vol. 100, no. 5, pp. 534-541, 2002. 

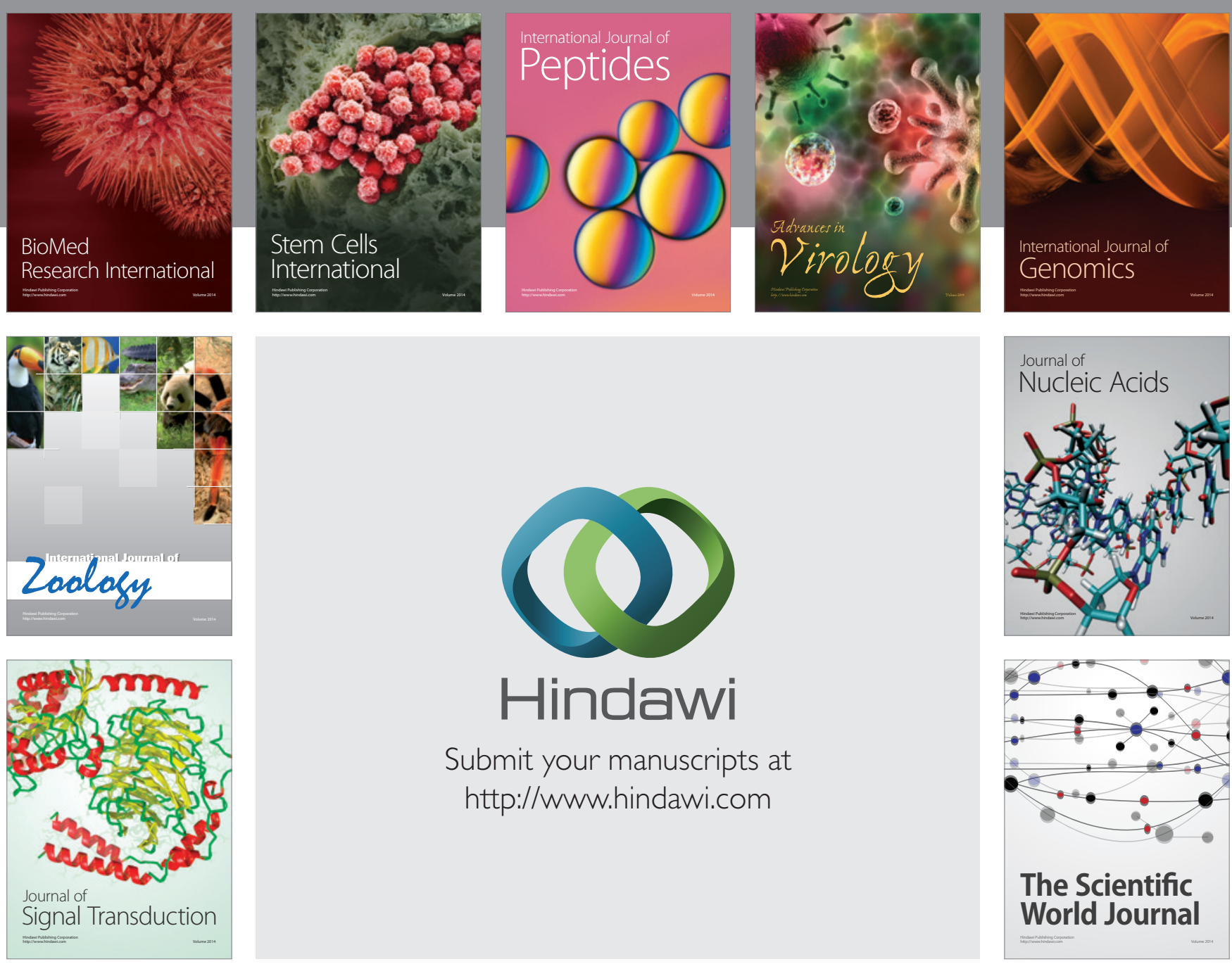

Submit your manuscripts at

http://www.hindawi.com
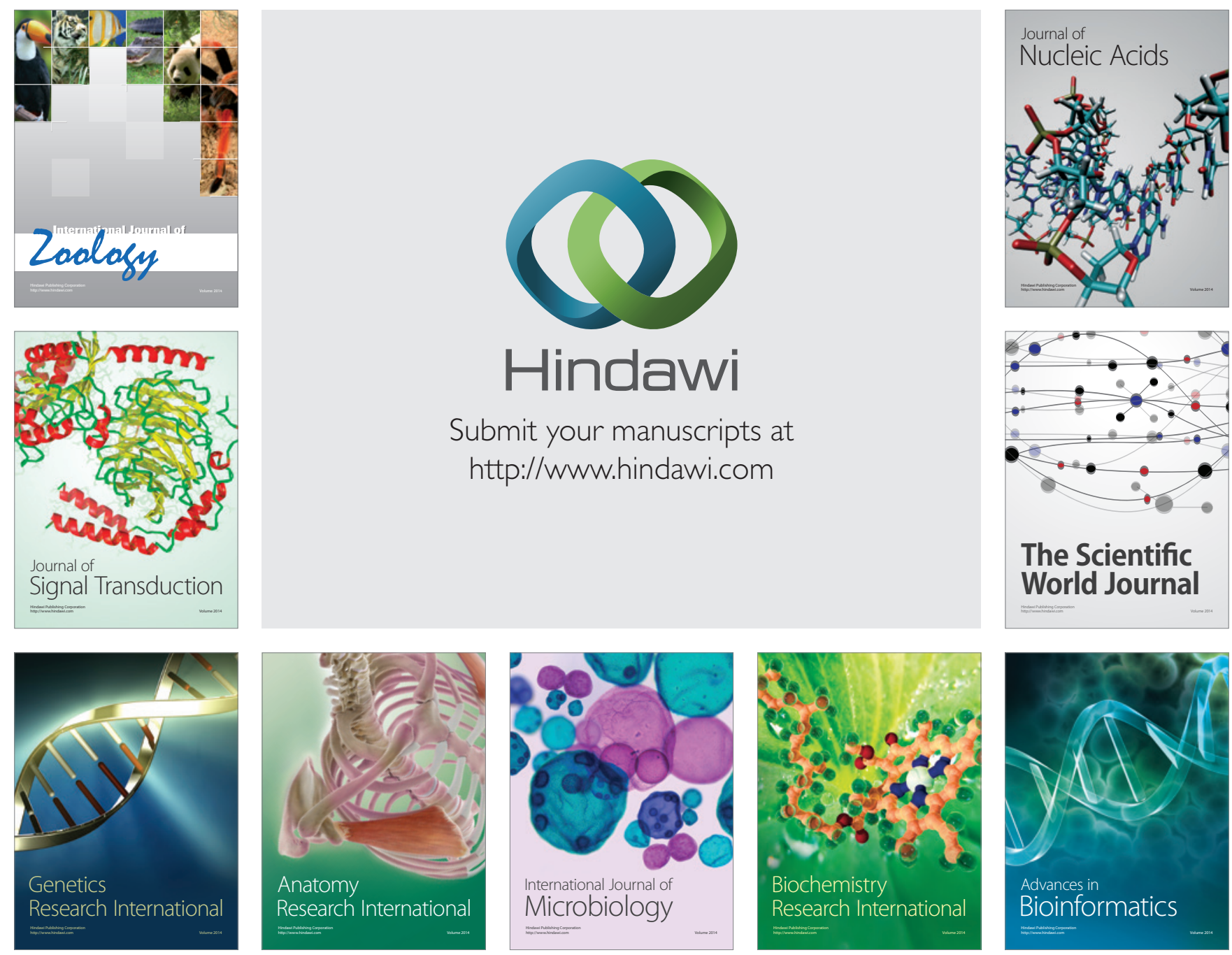

The Scientific World Journal
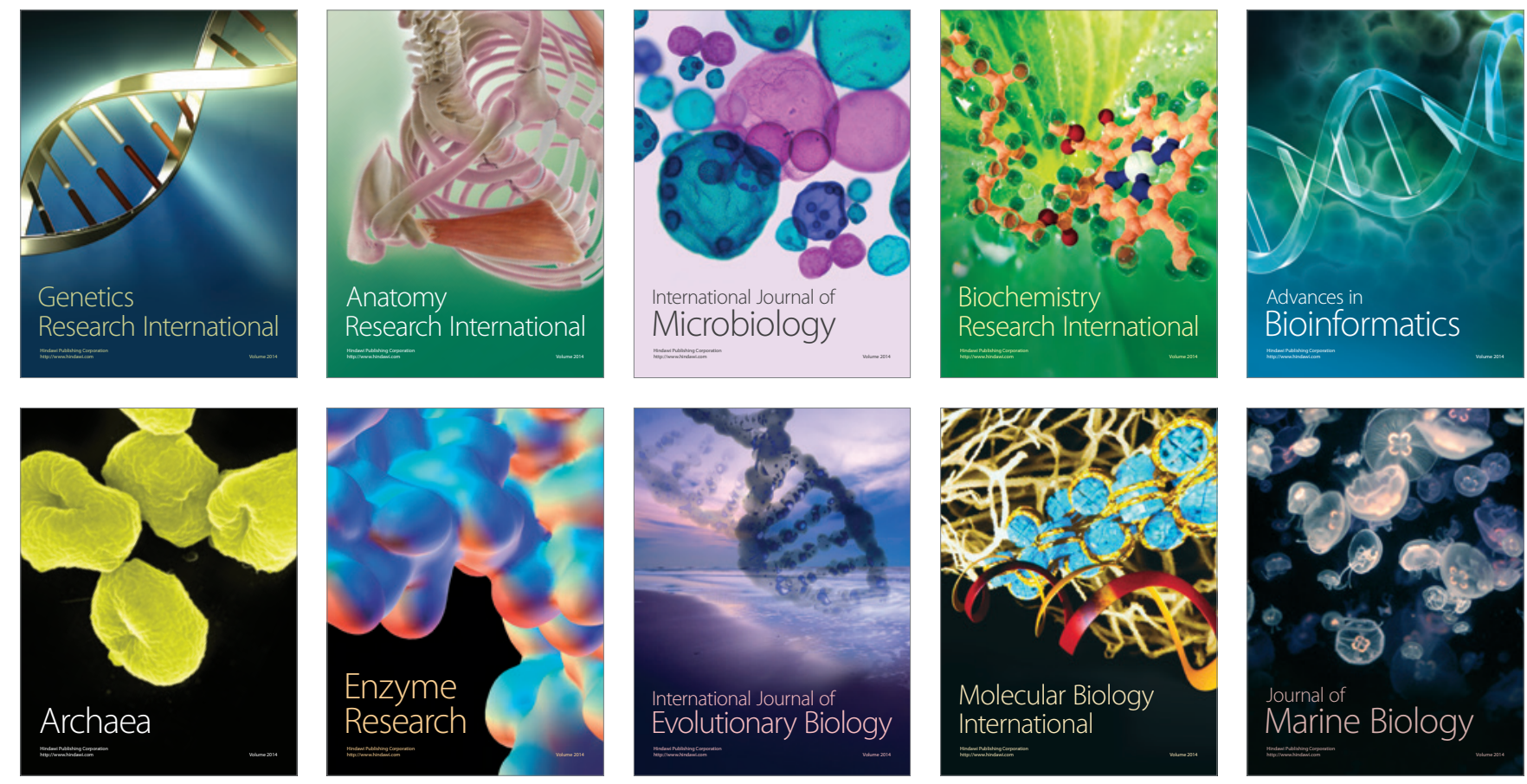